\title{
Clinical significance of STAT3 and MAPK phosphorylation, and the protein expression of cyclin D1 in skin squamous cell carcinoma tissues
}

\author{
JIAN WU ${ }^{1}$, WEN-YING LU ${ }^{2}$ and LEI-LEI CUI ${ }^{1}$ \\ ${ }^{1}$ Department of Laboratory Medicine, The First People's Hospital of Yancheng City, Yancheng, Jiangsu 224005; \\ ${ }^{2}$ Department of Pathology, The Sixth People's Hospital of Yancheng City, Yancheng, Jiangsu 224000, P.R. China
}

Received February 12, 2015; Accepted September 29, 2015

DOI: $10.3892 / \mathrm{mmr} .2015 .4460$

\begin{abstract}
The present study aimed to investigate the significance of the phosphorylation of signal transducer and activator of transcription 3 (STAT3) and mitogen-activated protein kinase (MAPK), and the protein expression of cyclin D1, in skin squamous cell carcinoma (SCC) tissues. SCC specimens from the skin were collected from 30 patients, and normal skin tissues were collected from 10 individuals as a control. Immunohistochemistry was used to assess the protein expression levels of phosphorylated (p-)STAT3, p-MAPK and cyclin D1 in the SCC tissues. The levels of p-STAT3 protein were abnormally increased in SCC $(\mathrm{P}<0.05)$; however, no significant differences in the protein expression of p-MAPK were identified between the normal skin and the SCC specimens. The extent of the upregulation of the expression of p-STAT3 and cyclin D1 correlated with the depth of tumor invasion $(\mathrm{P}<0.05)$. A positive correlation existed between the expression of p-STAT3 and cyclin D1 in SCC. However, no association between the expression intensity of p-MAPK and cyclin D1 was identified in SCC. It is postulated that the activation of STAT3 may induce the overexpression of cyclin D1, which results in the persistent proliferation of these tumor cells in SCC.
\end{abstract}

\section{Introduction}

Skin squamous cell carcinoma (SCC) occurs with a higher rate of incidence compared with several other malignant skin tumor types, and it occupies the largest percentage of the total skin malignant tumors (routinely, $80-90 \%)(1,2)$. The rate of incidence has increased on a year-by-year basis, particularly

Correspondence to: Mr. Wen-Ying Lu, Department of Pathology, The Sixth People's Hospital of Yancheng City, 66 Zhong Ting Road, Yancheng, Jiangsu 224000, P.R. China

E-mail: wujianglinxing@163.com

Key words: skin squamous cell carcinoma, STAT3, MAPK, phosphorylation, cyclin D1 among the elderly. The formation and development of the tumor occurs progressively, and is governed by multistep processes, involving the comprehensive action of internal and external factors $(3,4)$. In the last few years, research efforts have intensified in investigating the regulation of the disordering of fragmentation of cell proliferation, and the association which exists between cell signal transmission and the occurrence and development of tumors.

As two typical signaling molecules, which are involved in signaling cascades, signal transducer and activator of transcription 3 (STAT3) and mitogen-activated protein kinase (MAPK) are involved in the physical processes of cell growth, differentiation, division and development, and exert an important role in the malignant transformation of cells (5-7). STAT3 is an essential member of the STAT family of proteins. STAT3 is located in the cytoplasm in an unstimulated state, and upon stimulation, the structural SH2 domain interacts with a phosphorylated tyrosine residue, which itself is phosphorylated by Janus kinase (JAK).

In providing the axis for several types of signaling pathways, MAPK-cascaded activation exerts an important role in receiving signals, which are transferred and carried by membrane receptors and brought into the nucleus, which is an essential process for numerous signaling pathways associated with cell proliferation.

The overexpression of cyclin D1, which is one of the regulatory factors involved in the cell cycle, is a characteristic of numerous types of human primary tumor, and it is vitally important for the prognostication and the diagnosis of tumors $(8,9)$.

To investigate whether the growth of skin SCC, as with the majority of tumor types, comprises a cascade of molecular abnormalities whereby the regulation of the proliferation of the epidermal cells breaks down, and malignant transformation occurs $(10,11)$, immunohistochemical staining techniques were used to assess the phosphorylation of STAT3 and MAPK. Additionally, the protein levels of cyclin D1 were assessed, measured against normal skin tissue as a control, and the associations between phosphorylated (p-)STAT3, p-MAPK and cyclin D1 were investigated. The present study also aimed to explore the mechanism underlying skin SCC and to identify novel means by which an early diagnosis of the tumor may be accomplished. 
Table I. Positive staining results of EnVision ${ }^{\text {TM }}$ immunohistochemistry for p-STAT-3 in SCC and normal skin.

\begin{tabular}{|c|c|c|c|c|c|c|c|}
\hline \multirow[b]{2}{*}{ Group } & \multirow[b]{2}{*}{$\mathrm{n}$} & \multicolumn{3}{|c|}{ p-STAT3 positive } & \multirow[b]{2}{*}{ Negative (-) } & \multirow[b]{2}{*}{ PR (\%) } & \multirow[b]{2}{*}{ SPR (\%) } \\
\hline & & + & $2+$ & $3+$ & & & \\
\hline Normal & 10 & 0 & 0 & 0 & 10 & 0.0 & 0.0 \\
\hline SCC & 30 & 4 & 9 & 13 & 4 & 86.7 & 43.3 \\
\hline I & 8 & 1 & 2 & 2 & 3 & 62.5 & 25.0 \\
\hline II & 12 & 1 & 5 & 5 & 1 & 91.7 & 41.7 \\
\hline III & 10 & 2 & 2 & 6 & 0 & 100.0 & 60.0 \\
\hline
\end{tabular}

The $\chi^{2}$ test was used to compare the positive staining results between either the normal and SCC tissues, or between the SCC I and the SCC II or III group tissues $(\mathrm{P}<0.05)$. SCC, squamous cell carcinoma; PR, positive result; SPR, strong PR; SCC, squamous cell carcinoma; p-STAT3, phosphorylated signal transducer and activator of transcription-3.

\section{Materials and methods}

Patients and controls. Over the course of 5 years, samples of skin SCC were collected from 30 patients who received surgical resection in The First People's Hospital of Yancheng City (Yancheng, China), and who had been diagnosed by pathological confirmation. Each case had detailed clinical and pathological data, and no patient had received preoperative chemotherapy or radiotherapy. The patients with cancer included 20 males and 10 females,

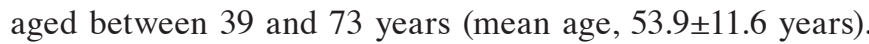
According to Broders' pathological grading criteria for skin SCC (12), eight cases were classified as grade I, 12 as grade II and 10 as grade III. Normal tissue specimens were collected by surgical resection from 10 individuals to serve as a control group. These included five males and five females, aged between 35 and 69 years (mean age, $49.5 \pm 10.4$ years). No statistically significant differences were detected in age or gender between the two groups. All specimens were obtained following informed patient consent and were approved by the Ethics Committee of The First People's Hospital of Yancheng City [Identification no. HMU (Ethics) 20121103].

Immunohistochemical staining techniques. The EnVision ${ }^{\mathrm{TM}}$ staining immunohistochemical method (Dako, Carpinteria, CA, USA) was used to detect the distribution of p-STAT3, p-MAPK and cyclin D1. The immunohistochemical procedures were performed strictly in accordance with the manufacturer's instructions. The EnVision ${ }^{\mathrm{TM}}$ and 3,3'-diaminobenzidine (DAB) chromogenic reagent kits (Santa Cruz Biotechnology, Inc., Dallas, TX, USA) were used for immunohistochemical staining. All slice staining was performed under identical conditions. The tissue was sliced to a diameter of $4 \mu \mathrm{m}$ prior to dehydration at room temperature for $60 \mathrm{~min}$ and dewaxing (placed in xylene for $10 \mathrm{~min}$, replacement of xylene, and soaking for $5 \mathrm{~min}$ in anhydrous alcohol, $5 \mathrm{~min}$ in 95\% ethanol and $5 \mathrm{~min}$ in $75 \%$ ethanol), and the slices were subsequently antigen-repaired using $0.01 \mathrm{~mol} / 1$ citric acid (pH 6.0). Normal goat serum was dropped on to the slice and incubated for $10 \mathrm{~min}$ at room temperature, and subsequently the corresponding specific antibodies were added to the slice and incubated for $1.5 \mathrm{~h}$ at room temperature. The following antibodies were used: p-STAT3 (cat. no. SC1409; Santa Cruz
Biotechnology, Inc; 1:1,000) and p-MAPK (cat. no. SC1312; Santa Cruz Biotechnology, Inc.; 1:1,000). The slices were washed with phosphate-buffered saline (PBS) three times ( $3 \mathrm{~min} / \mathrm{wash}$ ). The secondary antibody was dropped on to the slice and incubated for $30 \mathrm{~min}$ at room temperature. The slices were colored with DAB, the nuclei were stained using hematoxylin (Cell Signaling Technology, Shanghai, China), and the slices were subsequently dehydrated with gradient ethanol, cleared by xylene (Dako North America, Inc., Carpinteria, CA, USA) and sealed with natural gum (Cell Signaling Technology, Inc.). The staining of each batch was accompanied by a positive control (with the known positive section reagent, which was provided by Dako North America, Inc.) and a negative control (where the corresponding specific antibody was replaced with PBS).

Yellow- or tan-colored staining taken up by the nucleus or tan-reactant particles, respectively, indicated a positive result. Four independent experiments were performed for random detection using an optical microscope (BH-2; Olympus, Hamburg, Germany) at a high magnification (x200). According to the degree of the positive staining and the percentage of tumor cells present, the criteria for judgment were as follows: (-), no expression identified (only a small quantity of cell shading was present in $<5 \%$ of the cells); (1+), a low level of expression was identified (5-29\% of the total cells were pale yellow, positively identified cells); (2+), moderate expression was identified (30-59\% of the total cells were yellow, positively identified cells); (3+), high expression was identified (tan-coloured, positively identified cells were present at a level $>60 \%$ ).

Statistical analysis. SPSS 13.0 statistical software (SPSS, Inc., Chicago, IL, USA) was used for the statistical analyses. The $\chi^{2}$ test was used to compare the distribution of p-STAT3, p-MAPK and cyclin D1 between normal and cancer tissues, and Spearman's rank correlation coefficient analysis was used to analyze how the distribution of p-STAT3, p-MAPK and cyclin D1 among the tissues was associated. $\mathrm{P}<0.05$ was considered to indicate a statistically significant difference.

\section{Results}

Distribution of p-STAT3, p-MAPK and cyclin D1 in the nuclei of SCC and normal skin as determined by the dye 
Table II. Positive staining positive results of EnVision ${ }^{\mathrm{TM}}$ immunohistochemistry for p-MAPK in the SCC and normal skin.

\begin{tabular}{|c|c|c|c|c|c|c|c|}
\hline \multirow[b]{2}{*}{ Group } & \multirow[b]{2}{*}{$\mathrm{n}$} & \multicolumn{3}{|c|}{ p-MAPK positive } & \multirow[b]{2}{*}{ Negative (-) } & \multirow[b]{2}{*}{$\operatorname{PR}(\%)$} & \multirow[b]{2}{*}{$\operatorname{SPR}(\%)$} \\
\hline & & + & $2+$ & $3+$ & & & \\
\hline Normal & 10 & 0 & 0 & 0 & 10 & 0.00 & 0.00 \\
\hline $\mathrm{SCC}$ & 30 & 1 & 2 & 1 & 26 & 13.33 & 3.33 \\
\hline I & 8 & 0 & 0 & 0 & 8 & 0.00 & 0.00 \\
\hline II & 12 & 1 & 1 & 0 & 10 & 16.67 & 0.00 \\
\hline III & 10 & 0 & 1 & 1 & 8 & 20.00 & 10.00 \\
\hline
\end{tabular}

The $\chi^{2}$ test was used to compare the positive staining results between either the normal and SCC tissues, or between the SCC I and the SCC II or III group tissues $(\mathrm{P}<0.05)$. PR, positive result; SPR, strong PR; p-MAPK, phosphorylated mitogen-activated protein kinase C; SCC, squamous cell carcinoma.

A

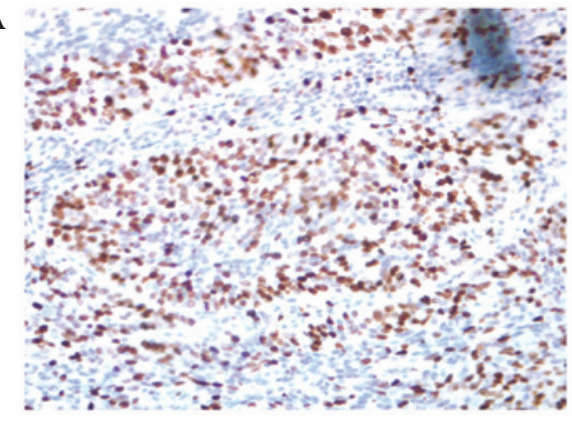

B

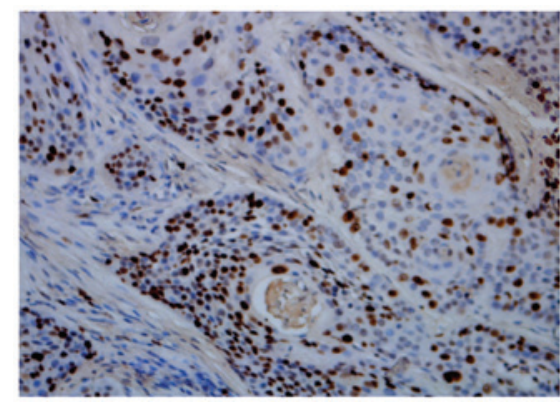

C

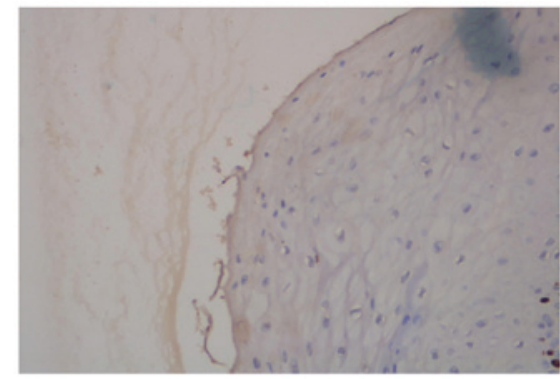

Figure 1. Representative images illustrating the results of the EnVision ${ }^{\mathrm{TM}}$ immunohistochemical analysis for p-STAT3 in SCC and in normal skin. The (A) markedly positive, (B) positive and (C) negative staining results for p-STAT3 in normal skin (magnification, x200). p-STAT3, phosphorylated signal transducer and activator of transcription 3; SCC, squamous cell carcinoma.

staining pattern. The positive result of p-STAT3 staining in the SCC tissues of $86.7 \%$ of the patients $(26 / 30)$ was significantly higher compared with the normal skin tissue $(0 ; \mathrm{P}<0.05)$. Furthermore, the staining intensity of $\mathrm{p}$-STAT3 in patients with SCC at the pathological grades II and III
A

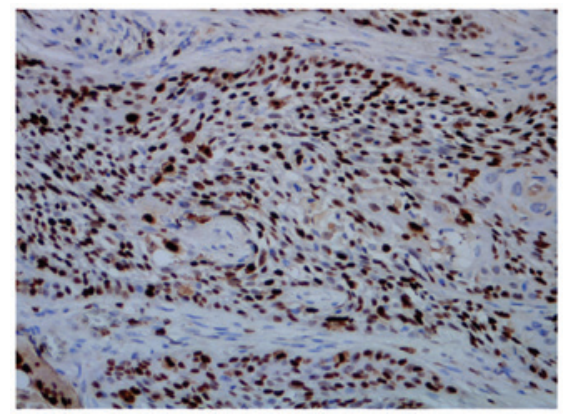

B

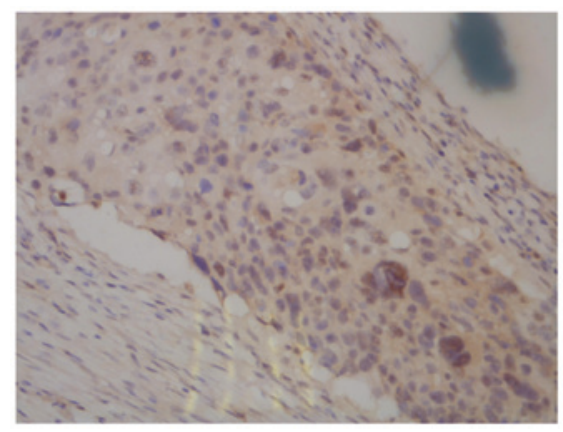

C

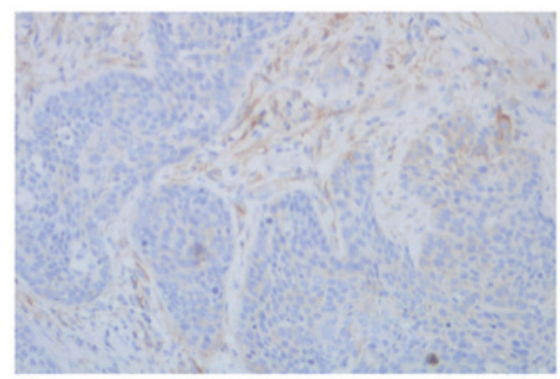

Figure 2. Representative images illustrating the results of the EnVision ${ }^{\mathrm{TM}}$ immunohistochemical analysis for p-MAPK in SCC. The (A) markedly positive, (B) positive and (C) negative staining results for p-MAPK in SCC (magnification, x200). p-MAPK, phosphorylated mitogen-activated protein kinase; SCC, squamous cell carcinoma.

was significantly higher compared with grade I $(\mathrm{P}<0.05$; Fig. 1A-C; Table I). A positive result was identified for p-MAPK in the SCC of $13.3 \%$, although it was revealed to be negative in normal skin tissues; in addition, no significant differences in the statistics were identified (Fig. 2A-C; 
Table III. Positive staining positive results of EnVision ${ }^{\mathrm{TM}}$ immunohistochemistry for cyclin D1 in the SCC and normal skin.

\begin{tabular}{|c|c|c|c|c|c|c|c|}
\hline \multirow[b]{2}{*}{ Group } & \multirow[b]{2}{*}{$\mathrm{n}$} & \multicolumn{3}{|c|}{ Cyclin D1 positive } & \multirow[b]{2}{*}{ Negative (-) } & \multirow[b]{2}{*}{ PR (\%) } & \multirow[b]{2}{*}{ SPR (\%) } \\
\hline & & + & $2+$ & $3+$ & & & \\
\hline Normal & 10 & 0 & 0 & 0 & 10 & 0.00 & 0.00 \\
\hline SCC & 30 & 3 & 8 & 11 & 8 & 73.33 & 36.67 \\
\hline I & 8 & 1 & 1 & 1 & 5 & 37.50 & 12.50 \\
\hline II & 12 & 1 & 4 & 5 & 2 & 75.00 & 41.67 \\
\hline III & 10 & 1 & 3 & 5 & 1 & 90.00 & 50.00 \\
\hline
\end{tabular}

The $\chi^{2}$ test was used to compare the positive staining results between either the normal and SCC tissues, or between the SCC I and the SCC II or III group tissues $(\mathrm{P}<0.05)$. SCC, squamous cell carcinoma; PR, positive result; SPR, strong PR.

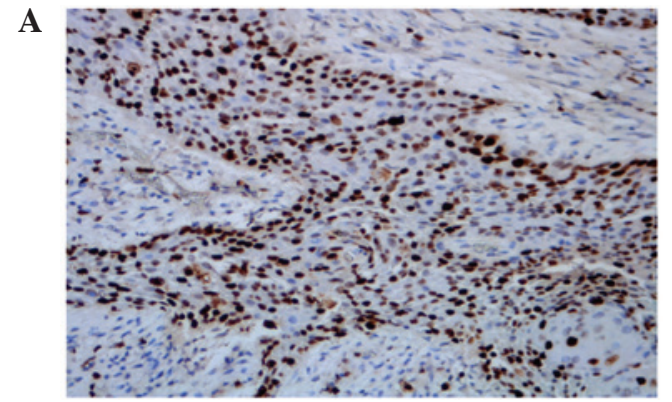

B

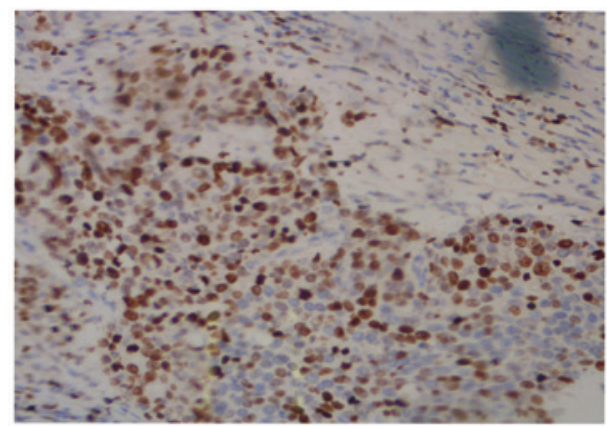

C

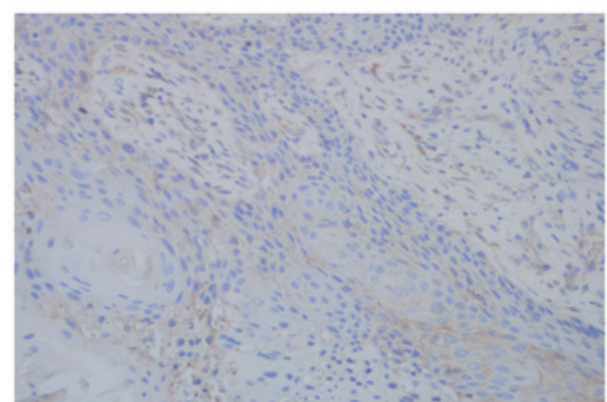

Figure 3. Representative images illustrating the results of the EnVision ${ }^{\mathrm{TM}}$ immunohistochemical analysis for cyclin D1 in SCC. The (A) markedly positive, (B) positive and (C) negative staining results for cyclin D1 in SCC (magnification, x200). SCC, squamous cell carcinoma.

Table II). Cyclin D1 protein was scattered throughout the epidermis and cancerous tissue, and the positive result of cyclin D1 in the SCC was determined to be $73.33 \%$ (22/30 patients). Furthermore, the positive staining result of cyclin D1 in the SCC at pathological grades II and III was significantly higher compared with grade $\mathrm{I}(\mathrm{P}<0.05$; Fig. 3A-C; Table III).
Table IV. Percentage staining results of p-STAT3, p-MAPK and cyclin D1 in skin squamous cell carcinoma.

\begin{tabular}{|c|c|c|c|}
\hline \multirow[b]{2}{*}{ Number } & \multicolumn{3}{|c|}{ Positive staining result (\%) } \\
\hline & p-STAT3 & p-MAPK & Cyclin D1 \\
\hline 1 & 50 & 5 & 5 \\
\hline 2 & 45 & 8 & 5 \\
\hline 3 & 85 & 10 & 2 \\
\hline 4 & 45 & 30 & 35 \\
\hline 5 & 23 & 12 & 20 \\
\hline 6 & 85 & 10 & 6 \\
\hline 7 & 45 & 65 & 8 \\
\hline 8 & 65 & 15 & 60 \\
\hline 9 & 53 & 4 & 3 \\
\hline 10 & 75 & 8 & 57 \\
\hline 11 & 50 & 7 & 5 \\
\hline 12 & 75 & 4 & 3 \\
\hline 13 & 49 & 5 & 3 \\
\hline 14 & 4 & 10 & 60 \\
\hline 15 & 45 & 8 & 30 \\
\hline 16 & 60 & 20 & 60 \\
\hline 17 & 58 & 35 & 30 \\
\hline 18 & 90 & 8 & 5 \\
\hline 19 & 50 & 8 & 4 \\
\hline 20 & 82 & 5 & 5 \\
\hline 21 & 40 & 75 & 15 \\
\hline 22 & 90 & 2 & 5 \\
\hline 23 & 18 & 90 & 50 \\
\hline 24 & 42 & 30 & 15 \\
\hline 25 & 80 & 10 & 10 \\
\hline 26 & 45 & 10 & 55 \\
\hline 27 & 50 & 70 & 8 \\
\hline 28 & 90 & 5 & 0 \\
\hline 29 & 42 & 12 & 30 \\
\hline 30 & 82 & 28 & 6 \\
\hline
\end{tabular}

p-STAT3, phosphorylated signal transducer and activator of transcription-3; p-MAPK, phosphorylated mitogen-activated protein kinase. 
Degree of correlation among the $p$-STAT3, $p$-MAPK and cyclin Dl proteins in SCC. To analyze the mutual associations among the proteins, according to the expression of each antigen in SCC, Spearman's correlation coefficient analysis was used. p-STAT3 and cyclin D1 were positively correlated with the intensity of positive staining $(r=0.714 ; \mathrm{P}<0.05)$, whereas the intensities of p-MAPK and cyclin D1 bore no positive correlation with the positive staining $(r=0.234$, $\mathrm{P}>0.05$; Table IV). Taken together, the results of the present study demonstrated that p-STAT3 protein was abnormally increased in SCC, however, no significant differences were observed in the protein expression levels of p-MAPK between normal skin and SCC. The positive rates of p-STAT3 and cyclinD1 were correlated with the depth of tumor invasion

\section{Discussion}

The occurrence and development of cancer is always closely associated with abnormalities in cellular signal transfer and regulation (13-15).

Several signaling pathways, which involve carcinogenic tyrosine kinases, converge onto STAT3, including those mediated by the epidermal growth factor receptor, interleukin-6/JAK and Src, and overactivation of these pathways occur in diverse types of cancer cells and tissues $(16,17)$. Following activation, STAT3 subsequently induces the anomalous expression of pivotal genes, which are crucial for various cellular activities, which consequently accelerate cell proliferation and a vicious transformation of the cells, and inhibits the various anticarcinogenic functions mediated by apoptosis; therefore, these genes are termed oncogenes $(18,19)$. The present study revealed that, compared with normal skin tissues, the positive results exhibited by the patients with SCC were indicative of clearly increased levels of p-STAT3, and the protein expression of STAT3 occurred in the nuclei in the case of SCC, suggesting that the abnormal activation of the signaling cascade mediated by STAT3 exerted an important role in the occurrence and development of skin SCC. The staining intensity of p-STAT3 for patients with skin SCC at the pathological grades II and III was notably increased, which suggested that the overactivation of STAT3 may be closely associated with the invasive growth of skin SCC.

MAPK remains in a static state in unstimulated cells, prior to being activated by the sequential activity of the protein kinases, MAPK kinase and MAP kinase kinase (20,21), which follows a sequential step-by-step phosphorylation process in cells stimulated by growth factors, among other stimulatory agents. Upon activation, MAPK is transferred into the nucleus and subsequently activates a target oncogene to stimulate cellular proliferation, and apoptosis is thereby inhibited. The results of the present study demonstrated a positive result of $13.3 \%$ for MAPK in skin SCC tissues, although this is statistically insignificant compared with the normal skin tissues, suggesting that the MAPK signal transduction pathway may not be the most important pathway in skin SCC.

Previous studies have indicated that the overexpression of cyclin D1 may result in a decrease in the G1 mitotic growth phase of the cells $(22,23)$, pushing them into the phase of synthesis, thereby completing the duplication of DNA. The increase in the protein expression of cyclin D1 is typically observed in certain primary malignant tumor types, including those of parathyroid adenoma, neck squamous cell carcinoma, breast cancer, esophageal cancer and hepatocellular carcinoma (24-28). Due to the fact that the expression of STAT3 and MAPK occurs upstream of the gene expression of cyclin D1, the expression of p-STAT3 in skin SCC increased directly in proportion with the positive intensity of cyclin D1. The results in the present study also revealed that the positive result of cyclin D1 in skin SCC was clearly higher compared with that in normal skin tissues, and the staining intensity of cyclin D1 in the case of skin SCC at pathological grades II and III was higher compared with that in grade I. These results suggested that there may an overexpression of cyclin D1 induced by the p-STAT3-mediated signal transduction pathway, leading to an increased level of cell proliferation in these tumor cells.

In conclusion, the present study has investigated the extent in which p-STAT3, p-MAPK and cyclin D1 were stained positively in skin SCC tissues compared with normal skin tissues. The association among the staining patterns were explored, with a view to characterizing a biological norm for diagnosing and prognosticating patients with cutaneous SCC. By disrupting the p-STAT3 signal transduction pathway in cutaneous SCC, the effect of p-STAT3, which leads to the overexpression of cyclin D1, on cutaneous SCC may be efficiently prevented. These results may be useful for future strategies of clinical drug antitumor therapy, and may provide the groundwork for a novel and pivotal method in this field.

\section{References}

1. Kraljik N, Rosso M, Ageel A, Sepić T and Gmajnić R: The incidence of skin squamous cell carcinoma in Osijek-Baranja County - an epidemiological study. Coll Antropol 35 (Suppl 2): 77-80, 2011.

2. Wu J, Zhang JR and Qin J: Methylation of E-cadherin and p14ARF gene promoters. Int J Clin Exp Med 7: 1808-1812, 2014.

3. Saladi RN, Nektalova T and Fox JL: Induction of skin carcinogenicity by alcohol and ultraviolet light. Clin Exp Dermatol 35: 7-11, 2010.

4. Gariboldi M, Peissel B, Fabbri A, Saran A, Zaffaroni D, Falvella FS, Spinola M, Tanuma J, Pazzaglia S, Mancuso MT, et al: SCCA2-like serpins mediate genetic predisposition to skin tumors. Cancer Res 63: 1871-1875, 2003.

5. Chen J, Chi M, Chen C and Zhang XD: Obesity and melanoma: Exploring molecular links. J Cell Biochem 114: 1955-1961, 2013.

6. Riebe C, Pries R, Schroeder KN and Wollenberg B: Phosphorylation of STAT3 in head and neck cancer requires p38 MAPKinase, whereas phosphorylation of STAT1 occurs via a different signaling pathway. Anticancer Res 31: 3819-3825, 2011.

7. Qu Y, Dang S and Hou P: Gene methylation in gastric cancer. Clin Chim Acta 424: 53-65, 2013.

8. Elliman SJ, Howley BV, Mehta DS, Fearnhead HO, Kemp DM and Barkley LR: Selective repression of the oncogene cyclin D1 by the tumor suppressor miR-206 in cancers. Oncogenesis 3: e113, 2014.

9. Liu X, Caffrey TC, Steele MM, Mohr A, Singh PK, Radhakrishnan P, Kelly DL, Wen Y and Hollingsworth, MA: MUC1 regulates cyclin D1 gene expression through p120 catenin and $\beta$-catenin. Oncogenesis 3, e107, 2014.

10. Evert M, Calvisi DF, Evert K, De Murtas V, Gasparetti G, Mattu S, Destefanis G, Ladu S, Zimmermann A, Delogu S, et al: Thymoma viral oncogene homolog/mammalian target of rapamycin activation induces a module of metabolic changes contributing to growth in insulin-induced hepatocarcinogenesis. Hepatology 55: 1473-1484, 2012. 
11. Molven A, Søvik O, von der Lippe C, Steine SJ, Njølstad PR, Houge $G$ and Prescott TE: Molecular genetic diagnostics in syndromes associated with the RAS/MAPK signalling pathway. Tidsskr Nor Laegeforen:129: 2358-2361, 2009.

12. Montagner A, Delgado MB, Tallichet-Blanc C, Chan JS, Sng MK, Mottaz H, Degueurce G, Lippi Y, Moret C, Baruchet M, et al: Src is activated by the nuclear receptor peroxisome proliferatoractivated receptor $\beta / \delta$ in ultraviolet radiation-induced skin cancer. EMBO Mol Med 6: 80-98, 2004.

13. Kim GT, Lee SH and Kim YM: Quercetin Regulates Sestrin 2-AMPK-mTOR Signaling Pathway and Induces Apoptosis via Increased Intracellular ROS in HCT116 Colon Cancer Cells. J Cancer Prev 18: 264-270, 2013.

14. Xu W, Yang Z, Zhou SF and Lu N: Posttranslational regulation of phosphatase and tensin homolog (PTEN) and its functional impact on cancer behaviors. Drug Des Devel Ther 8: 1745-1751, 2014.

15. Liang N, Zhang C, Dill P, Panasyuk G, Pion D, Koka V, Gallazzini M, Olson EN, Lam H, Henske EP, et al: Regulation of YAP by mTOR and autophagy reveals a therapeutic target of tuberous sclerosis complex. J Exp Med 211: 2249-2263, 2014.

16. Talbot JJ, Song X, Wang X, Rinschen MM, Doerr N, LaRiviere WB, Schermer B, Pei YP, Torres VE and Weimbs T: The cleaved cytoplasmic tail of polycystin-1 regulates Src-dependent STAT3 activation. J Am Soc Nephrol 25: 1737-1748, 2014

17. Gao SP, Mark KG, Leslie K, Pao W, Motoi N, Gerald WL, Travis WD, Bornmann W, Veach D, Clarkson B, et al: Mutations in the EGFR kinase domain mediate STAT3 activation via IL-6 production in human lung adenocarcinomas. J Clin Invest 117: 3846-3856, 2007.

18. Wang J, Zhang L, Chen G, Zhang J, Li Z, Lu W, Liu M and Pang X. Small molecule 1'-acetoxychavicol acetate suppresses breast tumor metastasis by regulating the SHP-1/STAT3/MMPs signaling pathway. Breast Cancer Res Treat 148, 279-289, 2014.

19. Snyder M,Huang J,Huang XY and Zhang JJA: A signal transducer

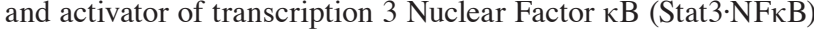
complex is necessary for the expression of fascin in metastatic breast cancer cells in response to interleukin (IL)- 6 and tumor necrosis factor (TNF)- $\gamma$. J Biol Chem 289: 30082-30089, 2014
20. Tian H, Zhang D, Gao Z, Li H, Zhang B, Zhang Q, Li L, Cheng Q, Pei D and Zheng J. MDA-7/IL-24 inhibits Nrf2-mediated antioxidant response through activation of $\mathrm{p} 38$ pathway and inhibition of ERK pathway involved in cancer cell apoptosis. Cancer Gene Ther 21: 416-426, 2014.

21. Casimiro MC, Wang C, Li Z, Di Sante G, Willmart NE, Addya S, Chen L, Liu Y, Lisanti MP and Pestell RG: Cyclin D1 determines estrogen signaling in the mammary gland in vivo. Mol Endocrinol 9: 1415-1428, 2013.

22. Pysz MA, Hao F, Hizli AA, Lum MA, Swetzig WM, Black AR, and Black JD: Differential regulation of cyclin D1 expression by protein kinase $C \alpha$ and $\varepsilon$ signaling in intestinal epithelial cells. J Biol Chem 289: 22268-22283, 2014

23. Nair SV, Ziaullah and Rupasinghe HP: Fatty Acid Esters of Phloridzin Induce Apoptosis of Human Liver Cancer Cells through Altered Gene Expression. PLoS One 9: e107149, 2014.

24. Zhang YJ, Jiang W, Chen CJ, Lee CS, Kahn SM, Santella RM and Weinstein IB: Amplification and overexpression of cyclin D1 in human hepatocellular carcinoma. Biochem Biophys Res Commun 29; 1010-1016, 1993.

25. Yang Y, Zhao LH, Huang B, Wang RY, Yuan SX, Tao QF, Xu Y, Sun HY, Lin C and Zhou WP: Pioglitazone, a PPAR $\gamma$ agonist, inhibits growth and invasion of human hepatocellular carcinoma via blockade of the rage signaling. Mol Carcinog 2014; [Epub ahead of print]

26. Shi QQ, Zuo GW, Feng ZQ, Zhao LC, Luo L, You ZM, Li DY, Xia J, Li J andChen DL: Effect of Trichostatin A on Anti HepG2 Liver Carcinoma Cells: Inhibition of HDAC Activity and Activation of Wnt $/ \beta$-Catenin Signaling. Asian Pac J Cancer Prev 15: 7849-7855, 2014

27. Wang X, Liu H, Wang X, Zeng Z, Xie LQ, Sun ZG and Wei MX: Preventive effect of Actinidia valvata Dunn extract on $\mathrm{N}$-methyl-N'-nitro-N-nitrosoguanidine-induced gastrointestinal cancer in rats. Asian Pac J Cancer Prev 15: 6363-6367, 2014.

28. Gopalakrishnan N, Saravanakumar M, Madankumar P, Thiyagu M and Devaraj H: Colocalization of $\kappa$-catenin with Notch intracellular domain in colon cancer: a possible role of Notch1 signaling in activation of Cyclin D1-mediated cell proliferation. Mol Cell Biochem 396: 281-293, 2014. 